\title{
Expected performance of the High-Energy Particle Detector on-board the second China Seismo-electromagnetic Satellite
}

\section{Francesco Maria Follega, ${ }^{a, b}$ Roberto luppa, ${ }^{a, b}$ Alberto Oliva, ${ }^{c}$ Michele Pozzato, ${ }^{c}$ Ester Ricci ${ }^{a, b}$ and Zouleikha Sahnoun ${ }^{c, *}$ on behalf of the CSES-Limadou \\ Collaboration}

(a complete list of authors can be found at the end of the proceedings)

${ }^{a}$ TIFPA - INFN Sez. of Trento,

Via Sommarive, 14 - 38123 Povo, Trento, Italy

${ }^{b}$ University of Trento,

Via Sommarive, 14 - 38123 Povo, Trento, Italy

${ }^{c}$ INFN Sez. of Bologna,

Viale C. Berti Pichat, 6/2 - 40127, Bologna, Italy

E-mail: francesco.follega@unitn.it, roberto.iuppa@unitn.it,

Alberto.0liva@bo.infn.it, michele.pozzato@bo.infn.it,

ester.ricci@unitn.it, sahnoun@bo.infn.it

The High Energy Particle Detector (HEPD) is one of the scientific payloads of the China SeismoElectromagnetic Satellite (CSES). The first satellite of the constellation was launched in February 2018 and has been operational in nominal conditions since then. With the launch of the CSES02 scheduled for mid 2022, the realization of the HEPD-02 detector is ongoing. The Limadou collaboration, in charge of the payload, updated the HEPD design to improve its performance. A Monte Carlo simulation has been developed using the GEANT4 tool, in order to study the response of the new detector to protons, electrons and light nuclei and validate the new design. The comparison between simulation results and data collected during tests will also allow to calibrate the detector response and to train a specifically designed neural network for event reconstruction. We report preliminary results from the simulation and show that the updated HEPD meets the scientific requirements of the CSES-02 mission

$37^{\text {th }}$ International Cosmic Ray Conference (ICRC 2021)

July 12th-23rd, 2021

Online - Berlin, Germany

\footnotetext{
*Presenter
} 


\section{Introduction}

The High Energy Particle Detector (HEPD-02) developed by the Italian CSES-LIMADOU collaboration is a particle detector of second generation to be flown on-board the CSES space mission [1, 2]. It is aimed to measure particle flux in the range $3-100 \mathrm{MeV}$ for electrons and $30-200 \mathrm{MeV}$ for protons, and correlate particle burst to the occurrence of strong seismic events and also to solar and cosmic phenomena. HEPD-02 is an update of the former HEPD-01 design flown on-board the CSES-01 satellite since February 2018. The new design is intended to allow measurements at lower energies. HEPD-02 is composed of several detectors:

- a first trigger plane (T1) made up of 5 plastic scintillator counters: $3.2 \times 15.4 \times 0.2 \mathrm{~cm}^{3}$.

- a direction detector (tracker) $15 \times 15 \mathrm{~cm}^{2}$ made of five standalone tracking modules, each composed of three sensitive planes.

- a second trigger plane (T2) segmented in orthogonal direction with respect to T1 and composed of 4 scintillators of dimensions $3.6 \times 15 \times 0.8 \mathrm{~cm}^{3}$.

- a range calorimeter made of a tower of 12 plastic scintillators $(\mathrm{P} 1, \ldots, \mathrm{P} 12), 15 \times 15 \times 1 \mathrm{~cm}^{3}$, and two orthogonal layers of 3 LYSO crystals: $5 \times 15 \times 4 \mathrm{~cm}^{3}$

- five plastic scintillator panels $0.8 \mathrm{~cm}$ thick, covering the sides and the bottom of the calorimeter are used as taggers.

All the scintillators are readout by 2 Hamamatsu R9880U-210 photomultiplier tubes (PMT). Details on the detector design can be found in [3,4]. In order to validate the new HEPD design and assess its capabilities a Monte Carlo simulation was developed. In the following are presented the preliminary results of the simulation and performance of the preliminary HEPD Qualification Model.

\section{Simulation}

A Monte Carlo simulation based on the GEANT4 toolkit [5] was developed in order to study the response of HEPD to electrons, protons and light ions and evaluate its performance. The full simulation takes a set of GDML files exported from the TCAD model of the detector. It includes the sensitive detectors (trigger, tracker, range detector, LYSO crystals and veto systems) and the mechanical structure and electronic box. In fig. 1 is presented the layout of the detector used in the simulation.

In the simulation the particle position, direction and energy release in each sensitive detector subsystem are recorded. In fig. 2 is shown the propagation and energy release in the sensitive detectors for vertical electrons with uniform initial kinetic energy distribution between 0 and $100 \mathrm{MeV}$. Simulation can also be run in optical mode, i.e. taking into account optical photon production, propagation and collection. The light emission spectra of plastic scintillators (EJ-200) and crystals (LYSO) together with the photomultiplier's photon to electrons conversion (quantum) efficiency were included. The reflectivity of the wrapping material and the material absorption coefficients are taken as parameters to be adjusted to best match experimental results. 


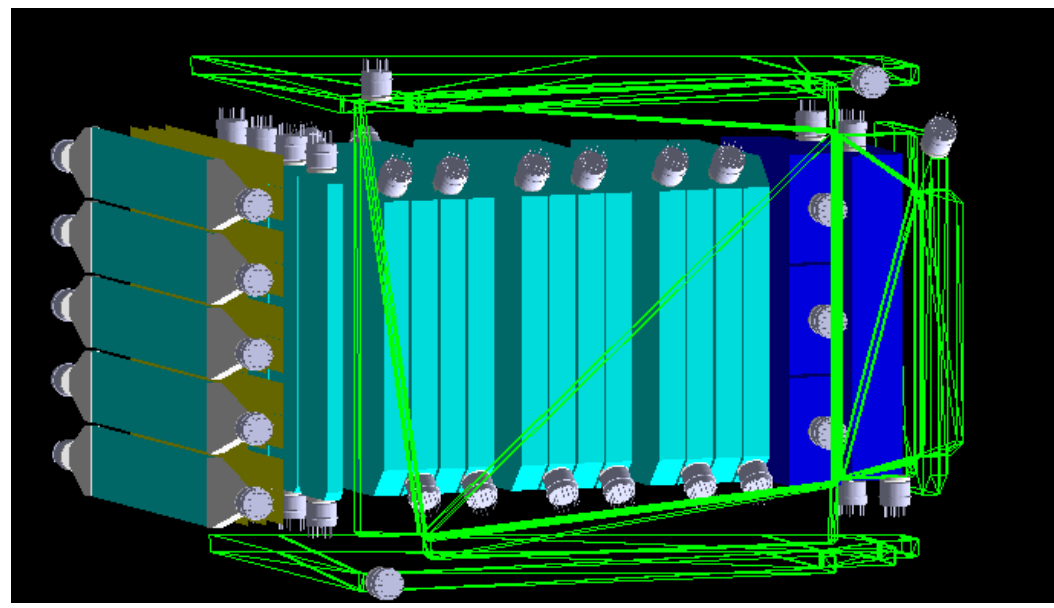

Figure 1: A detailed view of the detector sensitive parts. From left to right, the first trigger plane connected to PMTs through light-guides; the tracker system (dark yellow); the second trigger plane orthogonal to the first one; the 3 blocks plastic calorimeter tower and finally the 2 layers LYSO crystals (blue). All the plastic scintillators are in light-blue color. The containment panels are shown in transparency and have green outlines. Also shown in light-gray are the PMTs.
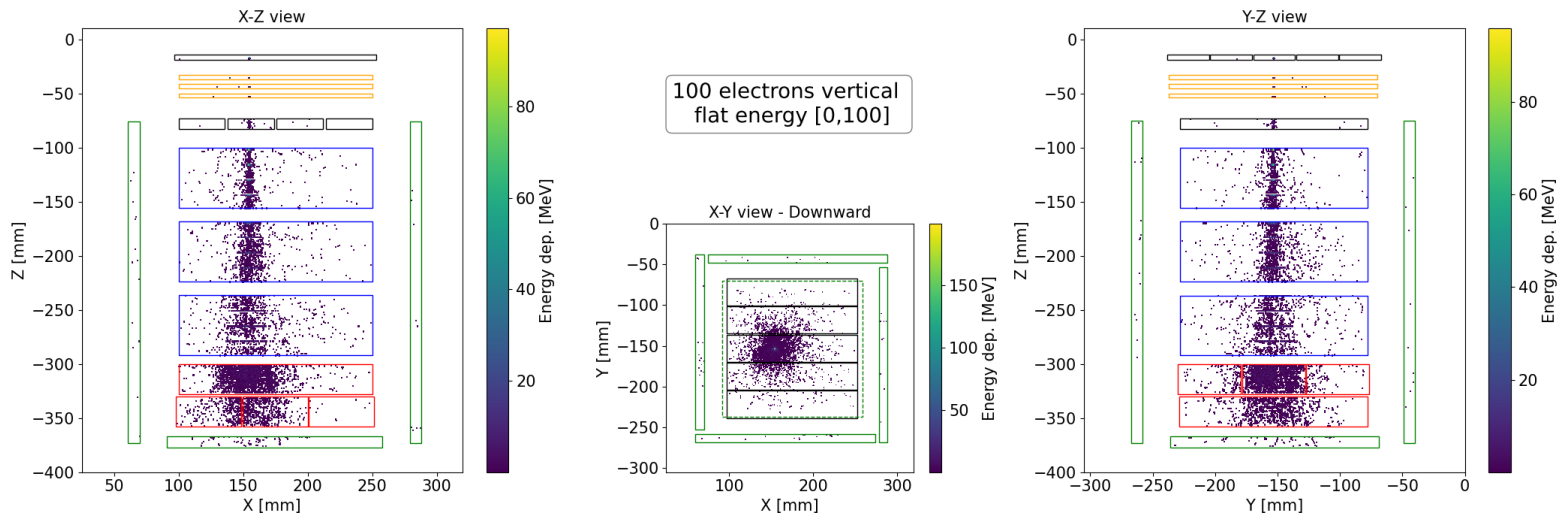

Figure 2: Location of the energy deposits in the sensitive detector volume of HEPD-01 generated by a distribution of vertical electrons with the same impinging trajectory and with a uniform energy distribution between 0 and $100 \mathrm{MeV}$. Three orthogonal projections are displayed.

\section{HEPD-02 scientific performance}

The study of HEPD performance has been carried out simulating three samples of particles: electrons, protons and alpha isotropically generated on top of the instrument with a uniform initial kinetic energy spectrum.

\subsection{Energy threshold}

Three trigger masks were examined, namely, $\mathrm{T} 1$ for which at least one bar of the first trigger plane is required to fire; T1\&T2; at least one bar of the first trigger plane and one bar of the second trigger plane are fired simultaneously; and finally, T1\&T2\&P1 where the first and second trigger 


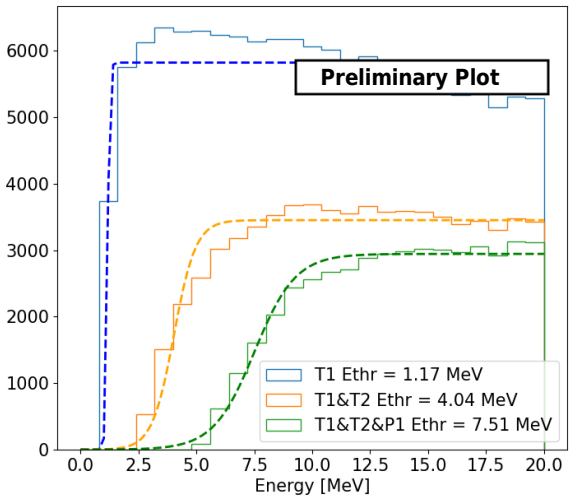

(a) Primary kinetic energy distribution of electrons.

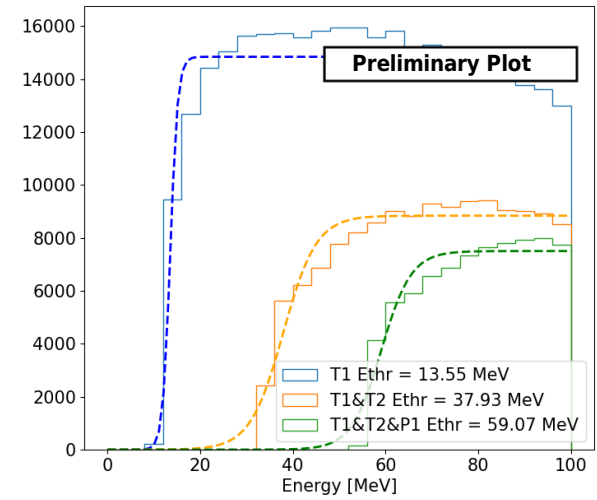

(b) Primary kinetic energy distribution of protons.

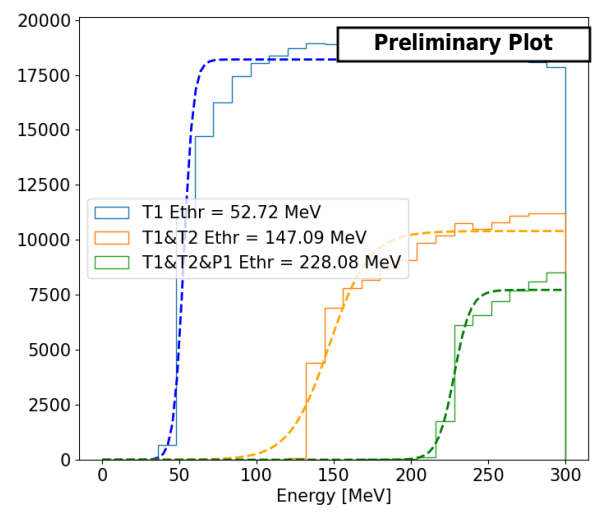

(c) Primary kinetic energy distribution of alpha particles.

Figure 3: The primary kinetic energy distribution of electrons, protons and alpha particles producing a trigger signal in different trigger configurations.

planes are required to fire in coincidence with the first calorimeter plane. In figs. $3 \mathrm{a}, 3 \mathrm{~b}$ and $3 \mathrm{c}$ are reported the preliminary results of the simulation. They show that a significant fraction of incoming electrons and protons reach at least the first trigger plane. Indeed, the minimum energies revealed in the case of $\mathrm{T} 1$ are $1.17 \mathrm{MeV}$ for electrons, $13.55 \mathrm{MeV}$ for protons and $52.72 \mathrm{MeV}$ for alpha particles. The use of a $2 \mathrm{~mm}$ thin trigger bar for the HEPD-02 T1 plane, while keeping a very high trigger efficiency [6], allows to reach lower energies than the first design HEPD-01 [2]

\subsection{Angular Resolution}

The angular resolution has been estimated on the integral simulation datasets and in bins of energy. The angular difference between the reconstructed track $\left(\overrightarrow{v e} c_{p r e d}\right)$ and the true direction $\left(v \vec{e} c_{\text {true }}\right)$ is defined as:

$$
\Psi=\arccos \left(v \vec{e} c_{\text {pred }} \cdot v \vec{e} c_{\text {true }}\right)
$$

By evaluating the angular reconstruction performance considering events in the T1\&T2 trigger configuration, one ensure to get the best achievable angular resolution by the tracker (the three 
tracker planes are hit). In fig.4a the distribution of $\Psi$ as a function of the energy is shown for protons.

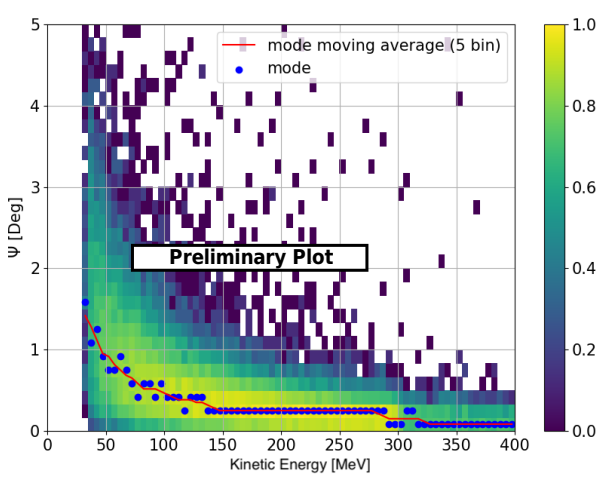

(a) Distribution of the angle $\Psi$ between the reconstructed incoming direction by the tracker and the true one for a sample of protons satisfying the T1\&T2 trigger configuration.

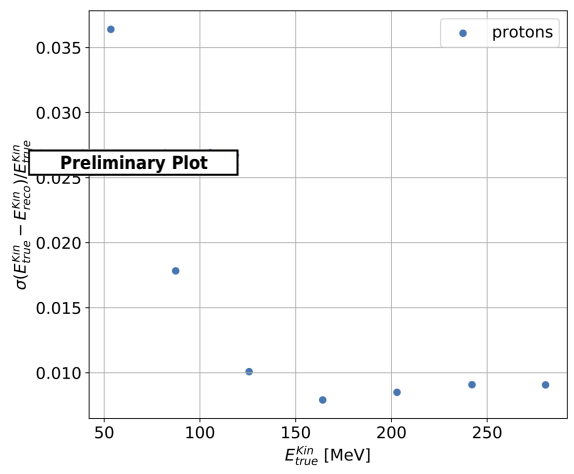

(b) Relative difference between true initial kinetic energy and reconstructed one, for a sample of protons satisfying typical event selection criteria.

Figure 4: The HEPD-02 detector angular and energy resolution.

\subsection{Energy resolution}

The particle initial energy is reconstructed from the energy deposited in each sensitive detector by training a Machine Learning algorithm called Random Forest Regressor [7, 8]. This algorithm is well-known in the among the multivariate analysis techniques. It consists in a meta estimator that fits an optimized number of decision trees, each on different sub-samples of the full dataset. After the fit it uses the average of all the tree to prevent over-fitting and improve the performance. It was trained on a dedicated MC sample of electron and protons to guarantee stable and reliable performance. This algorithm is able to reconstruct energies for electrons and protons. It automatically takes into account the profile of energy deposit compensating for the energy lost in inert materials such as covers or mechanical structure. In fig.4b are shown the performances of energy reconstruction tested with a statistically independent Monte Carlo proton sample.

\subsection{Particle Identification}

A similar algorithm called Random Forest Classifier [7, 9] was trained to discriminate along electron and protons and separate between electrons, protons and alpha particles. In fig.5 is shown an example of the achievable discrimination between electron, protons and He nuclei. In this case the algorithm exploits the different charge deposits of the various particles.

\section{Tuning MC to experimental data and Digitization strategy}

Preliminary experimental tests are being carried out in laboratory under cosmic muons in order to check for the efficiency and uniformity of the single counters. The tests are also very useful for a first tuning of the Monte Carlo simulation. As an example, in fig. 6 is given a preliminary 

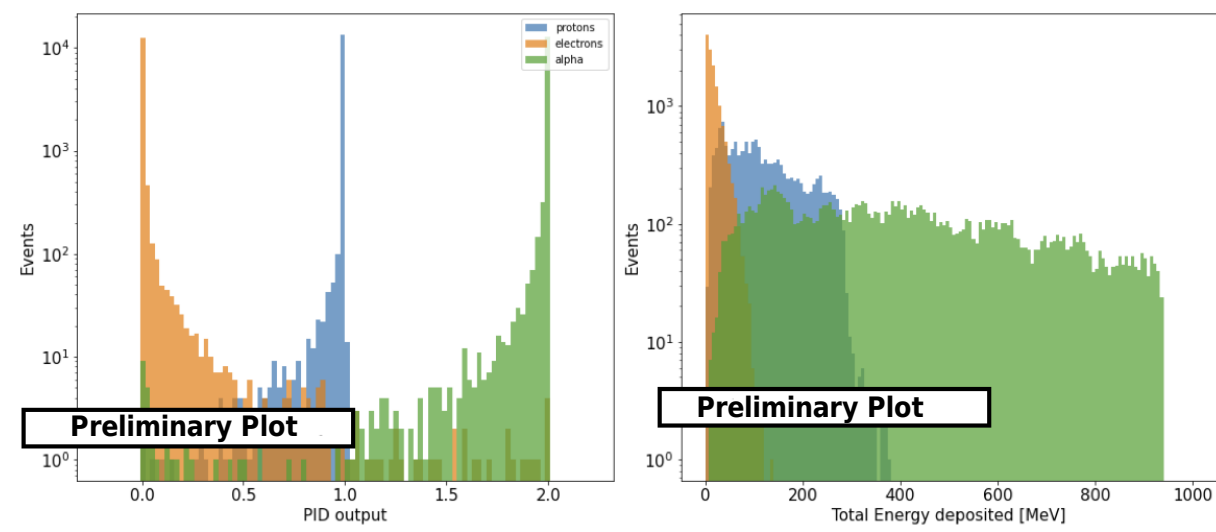

Figure 5: On the left the distribution of the PID output for electrons (orange), protons (blue) and alpha (green). On the right the distribution of the total energy deposited for the same particles.

comparison of the most probable number of photoelectrons registered by a single paddle of the first trigger plane (T1) with simulation as a function of the longitudinal impact position. The same comparison and tuning are to be done for the second trigger plane paddles and the calorimeter tiles and crystals. Running simulation with optical photons is very time consuming and CPU intensive, so a strategy is being developed in order to speed up the digitization process. It consists in building up 2D/3D maps of the mean number of photoelectrons relative to the center of the counter generated from a specific energy deposit at a specific position. These maps, combined with the distribution of the mean photoelectron number at the center of the counter as a function of the deposited energy and the corresponding ADC signal could be used for fast digitisation. An example map is shown in fig. 7 for photoelectrons collected by one of the phototubes of the T1 paddle, as a function on the impact position $(\mathrm{x}, \mathrm{y})$.

Next step should be the testing of the whole HEPD-02 apparatus under electron and ion beams at a Beam Test Facility.

\section{Conclusions}

In this paper were reported the results of the HEPD-02 detector simulation performed with the GEANT4 tool. The preliminary performances of the detector assuming an isotropic incoming flux of electrons, protons and alpha particles on top of the instrument, were also presented. First results show that HEPD-02 seems to meet expectations. Laboratory tests are underway to measure the performance of the prototypes, these results will be used to improve the MC description. 


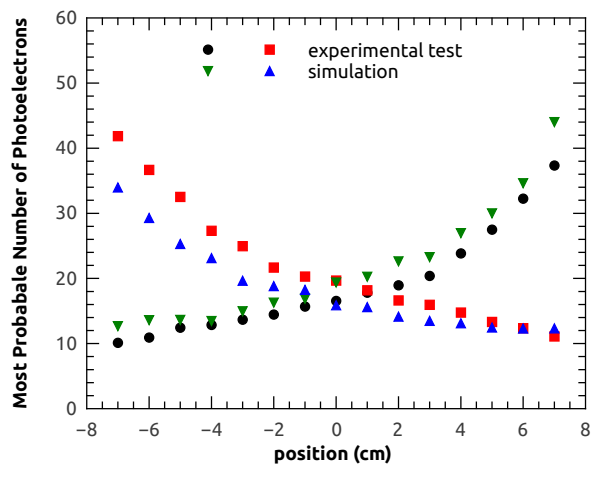

Figure 6: Comparison of the most probable number of photoelectrons obtained from simulation with experimental data as a function of longitudinal position for a T1 trigger paddle.

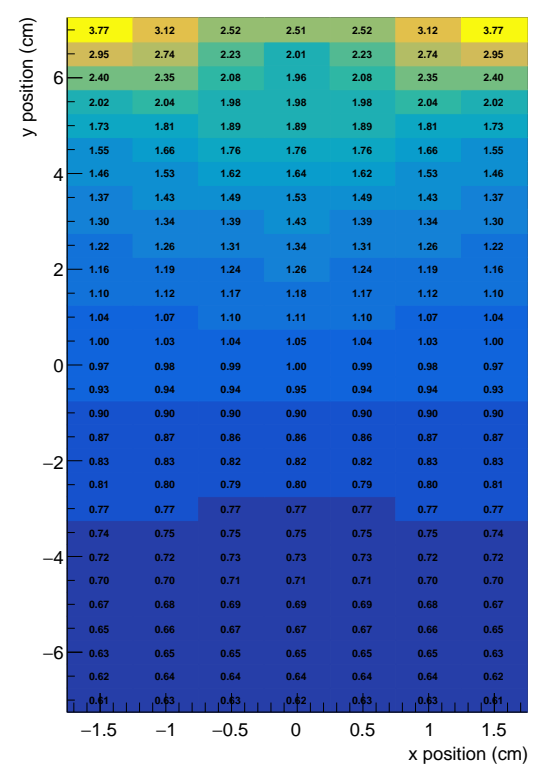

Figure 7: Map of the relative number of photoelectrons with respect to the center of the paddle for different interaction areas.

\section{Acknowledgments}

This work was supported by the Italian Space Agency in the framework of the agreement between ASI and INFN “Accordo Attuativo 2020-32.HH.0 Limadou Scienza+”.

\section{References}

[1] X. Shen, X. Zhang, S. Yuan et al., Sci. China Tech. Sci. 61 (2018) 634.

[2] P. Picozza, R. Battiston, G. Ambrosi et al., Ap. J. Supp. Ser. 243 (2019) 16

[3] A. Ambrosi, S. Bartocci, L. Basara et al., HEPD-02 QM Design Report, internal report RPT-LIM2-004-2.

[4] C. De Santis on behalf of the CSES-Limadou Collaboration, The High Energy Particle Detector (HEPD-02) for the second China Seismo-Electromagnetic Satellite (CSES-02), talk at the $37^{\text {th }}$ ICRC (2021).

[5] S. Agostinelli et al., Nucl. Instrum. Mehtods Phys. Res. A 506 (2003) 250

[6] A. Contin et al., Simulation and tests of HEPD-02 scintillator prototypes, talk given at the $106^{\text {th }}$ SIF Congress (2020), accepted for publication in Il Nuovo Cimento C

[7] Leo Breiman Random Forests, Machine Learning, 45(1), 5-32, 2001

[8] scikit-learn implementation of Random Forest Regressor

[9] scikit-learn implementation of Random Forest Classifier 


\section{Full Authors List: CSES-Limadou Collaboration}

S. Bartocci ${ }^{1}$, R. Battiston ${ }^{2,3}$, F. Benotto ${ }^{4}$, S. Beole ${ }^{4,5}$, W.J. Burger ${ }^{3,6}$, D. Campana ${ }^{7}$, G. Castellini ${ }^{8}$, P. Cipollone ${ }^{1}$, S. Coli ${ }^{4}$, L. Conti ${ }^{1,9}$, A. Contin ${ }^{10,11}$, M. Cristoforetti ${ }^{12}$, L. De Cilladi ${ }^{4,5}$, C. De Donato ${ }^{1}$, C. De Santis ${ }^{1}$, F.M. Follega ${ }^{2,3}$, G. Gebbia ${ }^{2,3}$, R. Iuppa ${ }^{2,3}$, M. Lolli $^{11}$, N. Marcelli ${ }^{1,13}$, M. Martucci ${ }^{1,13}$, G. Masciantonio ${ }^{1}$, M. Mergé ${ }^{1, \dagger}$, M. Mese $^{7,14}$, C. Neubuser ${ }^{3}$, F. Nozzoli ${ }^{3}$, A. Oliva ${ }^{11}$, G. Osteria $^{7}$, L. Pacini ${ }^{15}$, F. Palma ${ }^{1, \dagger}$, F. Palmonari ${ }^{10,11}$, A. Parmentier ${ }^{1}$, F. Perfetto ${ }^{7}$, P. Picozza ${ }^{1,13}$, M. Piersanti ${ }^{16}$, M. Pozzato ${ }^{11}$, E.

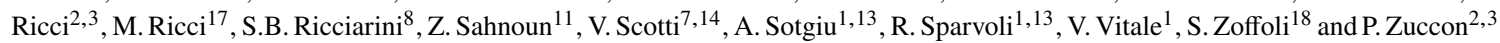

${ }^{1}$ INFN-Sezione di Roma “Tor Vergata”, V. della Ricerca Scientifica 1, I-00133 Rome, Italy;

${ }^{2}$ University of Trento, V. Sommarive 14, I-38123 Povo (Trento), Italy;

${ }^{3}$ INFN-TIFPA, V. Sommarive 14, I-38123 Povo (Trento), Italy;

${ }^{4}$ INFN-Sezione di Torino, Via P. Giuria 1, I-10125 Torino, Italy;

${ }^{5}$ University of Torino, Via P. Giuria 1, I-10125 Torino, Italy;

${ }^{6}$ Centro Fermi, V. Panisperna 89a, I-00184 Rome, Italy;

${ }^{7}$ INFN-Sezione di Napoli, V. Cintia, I-80126 Naples, Italy;

${ }^{8}$ IFAC-CNR, V. Madonna del Piano 10, I-50019 Sesto Fiorentino (Florence), Italy;

${ }^{9}$ Uninettuno University, C.so V. Emanuele II 39, I-00186 Rome, Italy;

${ }^{10}$ University of Bologna, V.le C. Berti Pichat 6/2, I-40127 Bologna, Italy;

${ }^{11}$ INFN-Sezione di Bologna, V.le C. Berti Pichat 6/2, I-40127 Bologna, Italy;

${ }^{12}$ Fondazione Bruno Kessler, V. Sommarive 18, I-38123 Povo (Trento), Italy;

${ }^{13}$ University of Rome "Tor Vergata", V. della Ricerca Scientifica 1, I-00133 Rome, Italy;

${ }^{14}$ University of Naples "Federico II", V. Cintia 21, I-80126 Naples, Italy;

15 INFN-Sezione di Firenze, V. Sansone 1, I-50019 Sesto Fiorentino (Florence), Italy;

${ }^{16}$ INAF-IAPS, V. Fosso del Cavaliere 100, I-00133 Rome, Italy;

17 INFN-LNF, V. E. Fermi 54, I-00044 Frascati (Rome), Italy;

${ }^{18}$ Italian Space Agency, V. del Politecnico, I-00133 Rome, Italy;

$\dagger$ At ASI Space Science Data Center (SSDC) also, V. del Politecnico, I-00133 Rome, Italy. 